\title{
openheart Nationwide prevalence and characteristics of transthyretin amyloid cardiomyopathy in Sweden
}

\author{
Rosa Elisabeth Lauppe (D) , ${ }^{1}$ Johan Liseth Hansen (D) , ${ }^{1,2}$ Christian Gerdesköld, ${ }^{3}$ \\ Mark H Rozenbaum, ${ }^{4}$ Anne Mette Strand, ${ }^{5}$ Merja Vakevainen, ${ }^{6}$ \\ Johanna Kuusisto (D) , Einar Gude, ${ }^{8}$ Finn Gustafsson, ${ }^{9}$ J Gustav Smith (1) ${ }^{10,11}$
}

\begin{abstract}
- Additional supplemental material is published online only. To view, please visit the journal online (http://dx.doi.org/10. 1136/openhrt-2021-001755).
\end{abstract}

To cite: Lauppe RE, Liseth Hansen J, Gerdesköld C, et al. Nationwide prevalence and characteristics of transthyretin amyloid cardiomyopathy in Sweden. Open Heart 2021;8:e001755. doi:10.1136/ openhrt-2021-001755

Received 10 June 2021 Accepted 23 September 2021

Check for updates

(c) Author(s) (or their employer(s)) 2021. Re-use permitted under CC BY-NC. No commercial re-use. See rights and permissions. Published by BMJ.

For numbered affiliations see end of article.

Correspondence to Dr J Gustav Smith; gustav. smith@med.lu.se

\section{ABSTRACT}

Objective Transthyretin amyloid cardiomyopathy (ATTR$\mathrm{CM}$ ) is a rare, progressive and fatal condition caused by deposition of transthyretin amyloid fibrils in the heart. This study aims to identify all patients diagnosed with ATTR-CM in Sweden, estimate the prevalence of ATTR-CM, describe patient characteristics and mortality, assess the importance of early symptoms (red flags) for identification of ATTR-CM, and compare with patients with heart failure (HF).

Methods This retrospective study combined multiple national health registers covering all specialist visits and prescriptions for the entire population of Sweden. Between January 2008 and December 2018, patients with ATTR$\mathrm{CM}$ were identified retrospectively based on a combination of diagnosis codes and compared with matched, all-cause non-ATTR HF patients.

Results Overall, a total of 994 patients diagnosed with ATTR-CM were identified, with an average age at diagnosis of 73 years, and $30 \%$ of whom were female. The prevalence of diagnosed ATTR-CM cases in 2018 was 5.0 per 100000 . The median survival from diagnosis was 37.6 months (Cl 33.8 to 43.8), with a lower median survival in women (27.9 months, $\mathrm{Cl} 23.3$ to 33.8) compared with men (43.5 months, $\mathrm{Cl} 37.6$ to 49.6). Patients with ATTR-CM demonstrated reduced survival compared with patients with HF $(p<0.001)$. Compared with patients with HF, clinical identification of carpal tunnel syndrome, spinal stenosis, and atrioventricular and left bundle branch block can facilitate earlier diagnosis of ATTR-CM.

Conclusions This study provides the first nationwide estimates of ATTR-CM prevalence and risk factors. The results reinforce the severity of the disease and the importance of earlier diagnosis, especially for female patients, in order to allow effective treatment and prevention of disease progression.

\section{INTRODUCTION}

Transthyretin amyloid cardiomyopathy (ATTR-CM) is a rare, progressive and fatal condition caused by deposition of the transthyretin amyloid fibrils in the heart. ATTR-CM can be hereditary, due to mutation in the transthyretin $(T T R)$ gene (variant ATTR or ATTRv), or associated with ageing

\section{Key questions}

What is already known about this subject?

- In recent years transthyretin amyloid cardiomyopathy (ATTR-CM) has received increased attention from physicians and scholars as the cause of heart failure (HF) and cardiomyopathy.

- Recent advances in disease-modifying treatments have made the recognition of ATTR-CM increasingly important to improve quality of life and mortality in a patient population with poor prognosis.

- Even though research on ATTR-CM has increased over the last years, large gaps remain in the knowledge of the epidemiology of ATTR-CM.

- Importantly, the prevalence of ATTR-CM in the population remains unclear.

- Patient characteristics, mortality and potential clinical findings (red flags) for ATTR-CM have been studied previously but were based on subgroups of the population, often covering shorter time periods, and have not previously been compared with all-cause non-ATTR HF patients.

(with a wild-type allelic constitution of the TTR gene, ATTRwt). ${ }^{1}$ Northern Sweden is known as an endemic area for ATTRv with a V30M mutation with a large population of late-onset cases, ${ }^{2}$ who have been found to more prominently develop cardiomyopathy compared with early-onset patients. ${ }^{3}$

Research suggests that many patients with ATTR-CM remain undiagnosed ${ }^{1}$ or are diagnosed with a delay of several years after symptom onset. ${ }^{4}$ Consequently, the prognosis for patients with ATTR-CM has been historically poor. Previous research shows a median survival of less than 4 years after diagnosis. ${ }^{5-7}$

The importance of early diagnosis has brought attention to clinical findings (red flags) which are associated with increased risk of ATTR-CM development. Although ATTR-CM commonly presents with symptoms of heart failure (HF) or arrhythmias, amyloidosis is a systemic disease and can cause 


\section{Key questions}

What does this study add?

- To our knowledge, this study provides the first nationwide estimates of ATTR-CM prevalence, mortality and risk factors.

- We combined multiple Swedish national registers to identify and study patients diagnosed with ATTR-CM.

- Sweden is well positioned for such nationwide studies, given full healthcare coverage, and mandatory reporting of diagnosis codes from all inpatient and outpatient specialist visits and drug prescriptions to nationwide registers.

- We also studied the occurrence of red flags associated with increased risk of ATTR-CM development that can help to facilitate early diagnosis of ATTR-CM.

- A look-back period of between 10 and 20 years for each patient made it possible to study red flags from nationwide registers long before the initial ATTR-CM diagnosis.

- To our knowledge our results are unique compared with previous studies as we were able to relate the prevalence of identified red flags in patients with ATTR-CM to patients with HF, and this enabled us to identify red flags that are unique to patients with ATTR-CM.

How might this impact on clinical practice?

Our results show that the median survival from diagnosis was just above 3 years.

- We compared the results with a matched population of patients with all-cause HF who had a median survival of 6 years, and interestingly the results also indicate a lower median survival (2.3 years) in female patients with ATTR-CM compared with male patients (3.6 years).

- This study provides insights into the characteristics of patients, mortality, red flags and severity of disease.

- The results reinforce the severity of disease and the importance of earlier diagnosis of ATTR-CM, especially for women.

- The insights into the red flags from this study can assist physicians in diagnosing patients with ATTR-CM earlier and potentially distinguishing them from the broader group of patients with HF.

various non-cardiac symptoms. Potential red flag diagnoses previously identified in the literature include carpal tunnel syndrome ${ }^{67}$ spinal stenosis, ${ }^{8}$ intestinal disorders, ${ }^{9}$ ruptured distal biceps tendon, ${ }^{10}$ as well as heart-related conditions such as aortic stenosis, ${ }^{11}$ atrial fibrillation and flutter, ${ }^{7}$ or atrioventricular block. ${ }^{12}$

Even though research on ATTR-CM has increased over the last years, large gaps remain in the knowledge of the epidemiology of ATTR-CM. Patient characteristics, mortality and potential red flags for ATTR-CM have been studied previously but were based on subgroups of the population, often covering shorter time periods, and have not previously been compared with non-ATTR HF patients. Sweden is well positioned for such nationwide studies, given full healthcare coverage, mandatory reporting of diagnosis codes from all inpatient and outpatient specialist visits and drug prescriptions to nationwide registers, and uniform use of personal identification numbers which allows linkage of registers.

The current study aimed to identify all patients with clinically diagnosed ATTR-CM in Sweden from national health registers, estimate the prevalence of ATTR-CM, and describe patient characteristics, mortality and the importance of red flags for identification of ATTR-CM in comparison with patients with HF.

\section{METHODS \\ Study design}

A retrospective cohort study was conducted using Swedish national population-based registers. Patients were identified between 1 January 2008 and 31 December 2018 and were followed until death or end of study period. For each patient, a minimum of 10 years look-back period was used to identify exclusion criteria, comorbidities and red flags.

\section{Data sources}

Patient-level data were extracted from the National Patient Register ${ }^{13}$ the Prescription Drug Register ${ }^{14}$ and the Cause of Death Register, ${ }^{15}$ and linked together using unique personal identifiers. The National Patient Register provides information on diagnoses according to the International Classification of Diseases version 10 (ICD-10), hospitalisations and outpatient specialist visits, as well as surgical and non-surgical procedures. The Prescription Drug Register contains data on all prescriptions filled at pharmacies, and the Cause of Death Register provides the confirmed dates of death and the registered cause of death. Due to mandatory reporting these national registers have a high degree of completeness. All data were obtained from the Swedish National Board of Health and Welfare, the data holder of the national registers used in this study. Data can be obtained from the Swedish National Board of Health and Welfare on approval from the Swedish Ethical Review Authority.

\section{Patient identification}

Patients were identified retrospectively based on a combination of diagnosis codes as there is no specific ICD code for ATTR-CM. An algorithm was developed to identify patients with ATTR-CM. Figure 1 describes the process of patient identification.

\section{Data extraction}

We first extracted all patients with an ICD-10 code for amyloidosis (AM) diagnoses (E85.0, E85.1, E85.2, E85.4, E85.8, E85.9), cardiomyopathy (CM) diagnoses (I42.0, I42.1, I42.2, I42.5, I42.8, I42.9, I43.1, I43.8) or HF diagnoses $(\mathrm{I} 50 *)$. The study population included all adult patients in Sweden with any of these diagnoses between 2008 and 2018.

\section{Identification of the ATTR-CM cohort}

From the study population, patients with ATTR-CM were identified. Patients with ATTR-CM were defined as individuals diagnosed with HF or CM and AM between 2008 and 2018. It was required that the HF/CM diagnosis and the AM diagnosis be not more than 2 years apart.

Several criteria were used to exclude patients with lightchain (AL) amyloidosis from this cohort, in addition to 


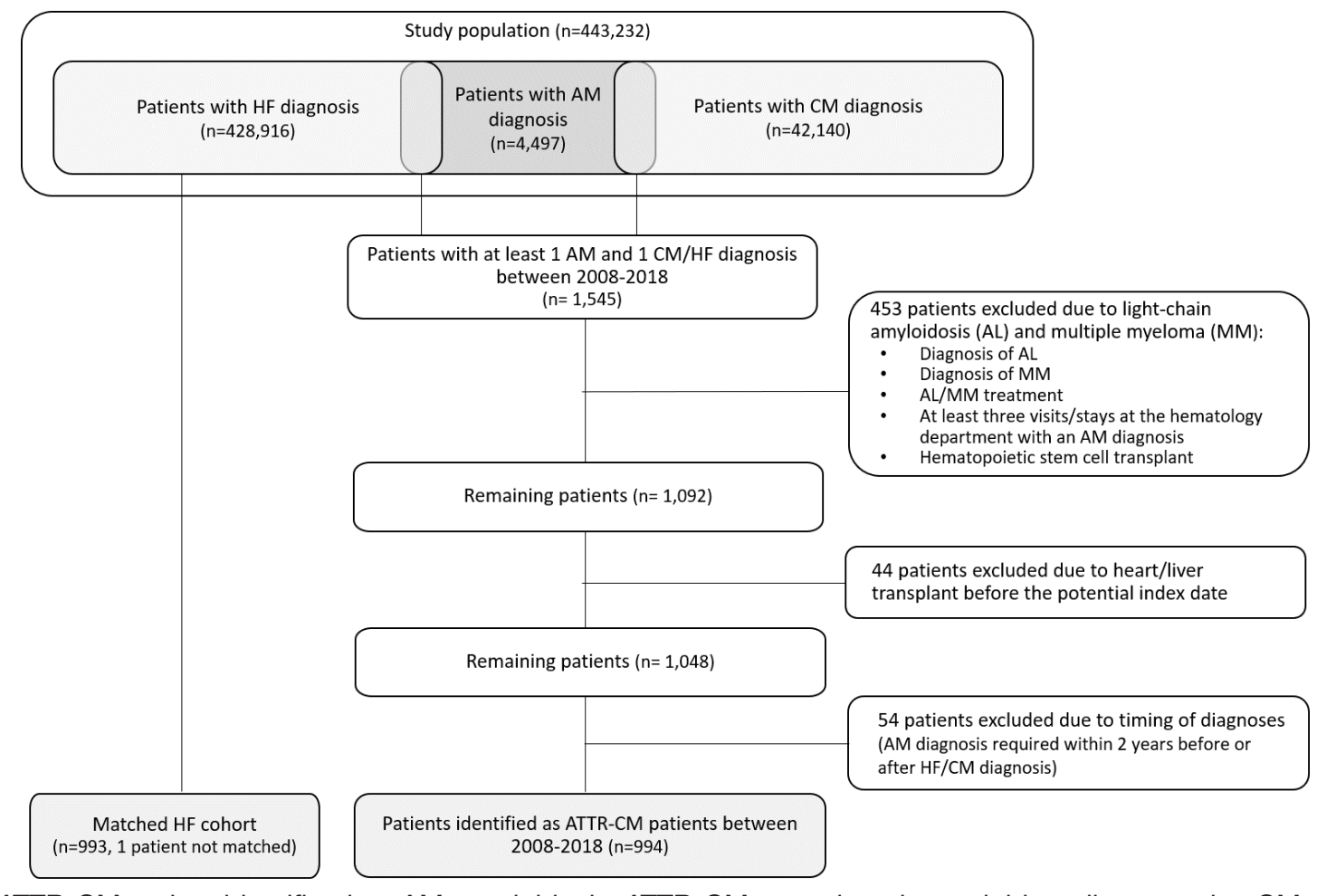

Figure 1 ATTR-CM patient identification. AM, amyloidosis; ATTR-CM, transthyretin amyloid cardiomyopathy; CM, cardiomyopathy; HF, heart failure.

exclusion based on the AL diagnosis code (ICD-10 code: E85.8A), which was introduced in Sweden only in 2014 and is not always used. Based on the association of AL amyloidosis with multiple myeloma $(\mathrm{MM}),{ }^{16}$ patients with MM diagnosis, patients with prescriptions of drugs commonly used in AL amyloidosis or MM treatment, and patients with haematopoietic stem cell transplant were also excluded. Moreover, patients with more than two AM diagnoses from the haematology department were defined as patients with AL amyloidosis and were excluded. In addition to patients with AL amyloidosis, individuals with a liver or heart transplant prior to diagnosis were excluded, as these are disease-modifying therapies.

The date of inclusion in the ATTR-CM cohort, the index date, was the date of the $\mathrm{CM} / \mathrm{HF}$ diagnosis used for identification. This date served as proxy for the patient's first ATTR-CM diagnosis and is referred to as the time of ATTR-CM diagnosis throughout the text.

\section{HF comparison cohort}

Patients with an HF diagnosis and not included in the ATTR-CM cohort were matched to patients in the ATTR-CM cohort. Patients were matched one-to-one, with replacement, on birth year, sex and the calendar year of diagnosis. The diagnosis date for patients in the matched HF cohort was the date of the first recorded HF diagnosis between 2008 and 2018.

\section{Statistical analyses}

All data management and statistical analyses were performed using R V.4.0. The t-test and proportion t-test were performed for continuous and binary outcomes, respectively. For time-to-event data log-rank tests were used. Mood's median test was performed for testing differences in median. The significance level used was $5 \%$ and CI is reported at the $95 \%$ level. As used in this text, the term 'average' refers to the mean.

\section{Patient characteristics}

Sex and age of the patients were measured at ATTR-CM diagnosis. Comorbidities were measured during 3 years before diagnosis; the Elixhauser Comorbidity Index ${ }^{17}$ with 31 categories $^{18}$ was used to measure the burden of comorbidity. Moreover, all pharmacy-dispensed prescriptions of heart or cardiovascular medication were recorded during 1 year before ATTR-CM diagnosis.

\section{Prevalence}

To estimate the prevalence in a certain year, the number of patients with ATTR-CM (patients alive at the beginning of the year plus the new cases diagnosed during that year) were divided by the Swedish population (number of residents on 31 December that year).

\section{Mortality}

Kaplan-Meier estimates and Cox proportional hazards regression were used to assess patients' survival after diagnosis compared with the matched control group.

\section{Red flags}

A descriptive analysis of the history of red flags up to the time of diagnosis was used to compare patients with ATTR-CM and the matched HF cohort; table 1 presents 
Table 1 History of potential red flag diagnoses and time from first occurrence of red flag to ATTR-CM diagnosis

\begin{tabular}{|c|c|c|c|c|c|}
\hline \multirow[b]{2}{*}{ Red flag diagnosis* } & \multicolumn{2}{|c|}{ Patients with ATTR-CM $(n=994)$} & \multicolumn{2}{|c|}{ HF comparison cohort $(n=993)$} & \multirow[b]{2}{*}{$\begin{array}{l}\text { P value; share of } \\
\text { patients } t \text {, median } \\
\text { years } \ddagger\end{array}$} \\
\hline & n (\%) & $\begin{array}{l}\text { Years from red } \\
\text { flag to index, } \\
\text { median }(q 25, q 75)\end{array}$ & n (\%) & $\begin{array}{l}\text { Years from red flag } \\
\text { to index, median } \\
\text { (q25, q75) }\end{array}$ & \\
\hline Carpal tunnel syndrome (unilateral and bilateral) & $167(16.8)$ & $6.7(3.7,10.6)$ & $32(3.2)$ & $6.4(3.0,9.7)$ & $0.023,0.636$ \\
\hline Spinal stenosis & $86(8.7)$ & $5.3(2.4,7.6)$ & $33(3.3)$ & $4.6(3.1,8.9)$ & $0.157,0.622$ \\
\hline Conductive and sensorineural hearing loss & $104(10.5)$ & $6.4(3.7,9.6)$ & $67(6.7)$ & $6.5(3.2,10.5)$ & $0.204,0.806$ \\
\hline Atrioventricular and left bundle branch block & $84(8.5)$ & $2.5(0.9,5.9)$ & $50(5.0)$ & $3.1(1.1,6.4)$ & $0.229,0.867$ \\
\hline Atrial fibrillation and flutter & $350(35.2)$ & $3.0(1.0,6.3)$ & $320(32.2)$ & $3.4(0.8,7.8)$ & $0.207,0.028$ \\
\hline Other functional intestinal disorders & $84(8.5)$ & $4.2(1.3,7.5)$ & $58(5.8)$ & $4.0(1.5,8.6)$ & $0.279,0.747$ \\
\hline Other cardiac arrhythmias & $82(8.2)$ & $5.6(2.2,10.4)$ & $61(6.1)$ & $3.1(0.5,6.3)$ & $0.316,0.671$ \\
\hline Other conduction disorders & $33(3.3)$ & $3.0(1.1,5.7)$ & $17(1.7)$ & $2.0(0.2,5.0)$ & $0.372,0.081$ \\
\hline Supraventricular tachycardia & $26(2.6)$ & $3.9(2.2,7.9)$ & $11(1.1)$ & $2.0(1.3,5.6)$ & $0.387,0.900$ \\
\hline Non-rheumatic aortic (valve) stenosis & $39(3.9)$ & $3.4(1.2,7.9)$ & $52(5.2)$ & $3.4(0.9,7.1)$ & $0.616,0.436$ \\
\hline Sick sinus syndrome & $27(2.7)$ & $6.7(4.3,10.9)$ & $21(2.1)$ & $2.2(0.3,4.7)$ & $0.447,0.604$ \\
\hline Other specified cardiac arrhythmias & $11(1.1)$ & $3.5(0.5,7.3)$ & $7(0.7)$ & $4.7(2.4,9.8)$ & $0.466,0.927$ \\
\hline Irritable bowel syndrome & $18(1.8)$ & $3.7(1.5,7.2)$ & $15(1.5)$ & $6.3(5.0,9.0)$ & $0.473,0.086$ \\
\hline $\begin{array}{l}\text { Injury of muscle and tendon of other parts of } \\
\text { biceps }\end{array}$ & $0(0.0)$ & - & $0(0.0)$ & - & - \\
\hline $\begin{array}{l}\text { Injury of muscle and tendon of long head of } \\
\text { biceps }\end{array}$ & $\leq 5$ & $11.9(10.3,13.5)$ & $0(0.0)$ & - & - \\
\hline Primary pulmonary hypertension & $\leq 5$ & $0.8(0.3,1.8)$ & - & $5.2(3.7,5.9)$ &,- 0.655 \\
\hline Other secondary pulmonary hypertension & $\leq 5$ & $6.0(5.0,6.9)$ & $\leq 5$ & $0.3(0.2,1.4)$ &,- 0.626 \\
\hline Acute pericarditis & $8(0.8)$ & $0.4(0.2,1.5)$ & $\leq 5$ & $0.2(0.1,4.2)$ &,- 0.791 \\
\hline
\end{tabular}

*Red flags are ordered after \% point difference between patients with ATTR-CM and patients with HF.

$\dagger P$ value for the difference between share of patients with each red flag in the ATTR-CM cohort and the HF comparison cohort (columns 2 and 4 ). $\ddagger \mathrm{P}$ value for the difference between median years from red flag to index in the ATTR-CM cohort and the HF comparison cohort (columns 3 and 5). ATTR-CM, transthyretin amyloid cardiomyopathy; HF, heart failure.

a full list of included red flag diagnoses. In addition, a multivariate logistic regression model was used to estimate the importance of the same red flags in predicting a later diagnosis of ATTR-CM. Variables included in the regression were the red flag diagnoses listed in table 1 , as well as age, sex and comorbidity index at diagnosis. The entire study population was included in the analysis and the outcome was whether patients were included in the ATTR-CM cohort or not.

\section{RESULTS \\ Patient identification}

The study population, consisting of all Swedish inhabitants who had at least one CM, HF or AM diagnosis after 2008, included 443231 individuals (see figure 1). Of the patients with an AM diagnosis $(\mathrm{n}=4497)$, more than a quarter $(n=1545)$ also had an HF or CM diagnosis. Of these patients, 497 were excluded as they were suspected to be patients with $\mathrm{AL} / \mathrm{MM}(\mathrm{n}=453)$ or had undergone a heart or liver transplant $(\mathrm{n}=44)$ before ATTR-CM diagnosis. Further, 54 patients were excluded as they did not fulfil the requirement regarding timing of the diagnoses. Finally, 994 patients were identified as patients with ATTR-CM during the period 2008-2018. Since one patient with ATTR-CM could not be matched, the matched HF cohort included 993 patients.

\section{Patient characteristics}

The mean age at diagnosis was 72.2 years and 72.9 years for women and men, respectively. Of the patients, $30 \%$ were female.

Patients with ATTR-CM had a mean Elixhauser Comorbidity Index of 5.13 (5.30 for HF cohort), indicating that patients on average received diagnoses in five disease categories in the 3 years before diagnosis. The five most common diagnoses were hypertension, cardiac arrhythmias, congestive heart failure, renal failure and diabetes. The average comorbidity index was slightly higher for female patients with ATTR-CM, with 5.64 compared with 4.91 for male patients.

A large majority (85\%) of patients with ATTR-CM and $\mathrm{HF}$ were treated with at least one $\mathrm{HF}$ and cardiovascular disease medication listed in table 2 in the year before identification. Relatively more patients of the ATTR-CM cohort were treated with diuretics compared with the matched patients with HF. Few patients in the ATTR-CM cohort $(n=27,2.7 \%)$ and none in the HF cohort were treated with diflunisal. No other disease-modifying treatments were used within the year before diagnosis. 


\begin{tabular}{|c|c|c|c|}
\hline & $\begin{array}{l}\text { Patients with ATTR-CM } \\
(\mathrm{n}=994)\end{array}$ & $\begin{array}{l}\text { HF comparison cohort } \\
\text { (matched) }(n=993)\end{array}$ & $P$ value \\
\hline Female, $n(\%)$ & $297(29.9)$ & $296(29.8)^{\star}$ & 0.493 \\
\hline Age at diagnosis, mean (SD) & $72.7(11.6)$ & $72.8(11.4)^{*}$ & 0.595 \\
\hline \multicolumn{4}{|l|}{ Age distribution at diagnosis, $\mathrm{n}(\%)$} \\
\hline $18-29$ & $7(0.7)$ & $6(0.6)$ & 0.491 \\
\hline $30-39$ & $11(1.1)$ & $10(1)$ & 0.491 \\
\hline $40-49$ & $33(3.3)$ & $31(3.1)$ & 0.482 \\
\hline $50-59$ & $69(6.9)$ & $75(7.6)$ & 0.556 \\
\hline $60-69$ & $176(17.7)$ & $173(17.4)$ & 0.472 \\
\hline $70-79$ & 400 (40.2) & 400 (40.3) & 0.505 \\
\hline $80+$ & $298(30)$ & $298(30)$ & 0.503 \\
\hline Elixhauser Comorbidity Index†, mean (SD) & $5.13(2.42)$ & $5.30(2.35)$ & 0.951 \\
\hline \multicolumn{4}{|c|}{ Prescriptions of heart and cardiovascular medication 1 year prior to diagnosis, $n(\%)$} \\
\hline Beta blockers & $584(58.8)$ & $596(60)$ & 0.671 \\
\hline ACE inhibitors & $382(38.4)$ & $369(37.2)$ & 0.360 \\
\hline ARBs & $292(29.4)$ & $233(23.5)$ & 0.064 \\
\hline Digoxin & $74(7.4)$ & $60(6)$ & 0.374 \\
\hline Dihydropyridine CCBs & $212(21.3)$ & $273(27.5)$ & 0.953 \\
\hline Non-dihydropyridine CCBs & $14(1.4)$ & $18(1.8)$ & 0.862 \\
\hline Diuretics & $608(61.2)$ & $446(44.9)$ & 0.000 \\
\hline Loop diuretics & 539 (54.2) & $381(38.3)$ & 0.000 \\
\hline Antiplatelets & 370 (37.2) & 438 (44.1) & 0.976 \\
\hline Lipid-lowering agents & $359(36.1)$ & 441 (44.4) & 0.991 \\
\hline Anticoagulants & 327 (32.9) & $303(30.5)$ & 0.260 \\
\hline
\end{tabular}

${ }^{*}$ Age and sex were used to match patients with HF to patients with ATTR-CM and are therefore very similar between the cohorts.

†The Elixhauser Comorbidity Index was calculated based on primary and secondary diagnoses in inpatient and outpatient specialty care. The index includes 31 diagnosis categories, each contributing 1 point to the index; the index can thus range from 0 to 31 .

ARBs, angiotensin II receptor blockers; ATTR-CM, transthyretin amyloid cardiomyopathy; CCBs, calcium channel blockers; HF, heart failure.

\section{Prevalence}

The mean prevalence of ATTR-CM in Sweden over the period of 2008-2017 was 2.9 per 100000 inhabitants, increasing to 5.0 per 100000 in 2018 (table 3). The prevalence in 2018 among men was 7.4 per 100000 compared with 2.5 per 100000 among women. On average, 90 patients were included in the cohort each year. The prevalence was highest in the Northern Swedish regions, as shown in figure 2. In the region of Västerbotten the prevalence in 2018 was 31.5 per 100000 and in Norrbotten 16.0 per 100000 compared with the country's average of 5.0 per 100000 .

\section{Mortality}

The median survival time for all patients with ATTR-CM after diagnosis was 37.6 months (CI 33.8 to 43.8). Overall, $77 \%$ (CI 77.4 to 79.7 ) of patients were alive 1 year after diagnosis, while the 5-year survival rate was $36.4 \%$ (CI 32.9 to 40.3). Patients with ATTR-CM demonstrated significantly higher mortality than the matched patients with HF ( $p<0.001$, HR: 0.60 , CI 0.53 to 0.68$)$. For the matched patients with HF, the median survival time after diagnosis was 72.7 months (CI 63.9 to 84.4 ) and the 5-year survival rate was $55.3 \%$ (CI 51.7 to 59.1). Women with ATTR-CM demonstrated higher mortality than men $(p=0.001$, HR: 0.75 , CI 0.63 to 0.90 ); the median survival time after diagnosis was 43.5 months (CI 37.6 to 49.6 ) for men and 27.9 months (CI 23.3 to 33.8) for women. In contrast, median survival in the HF cohort was 85.7 months (CI 64.4 to N/A; not sufficient events to estimate the $95 \%$ CI upper bound for median survival time) for women and 69.7 months (CI 60.1 to 82.5) for men ( $\mathrm{p}=0.056$, HR: 1.23, CI 0.99 to 1.52 ). The Kaplan-Meier curves for overall survival are shown in figure 3; confidence bands are reported at the $95 \%$ level.

\section{Red flags}

The largest differences between the share of patients with ATTR-CM and patients with HF with a history of red flag diagnoses were observed for carpal tunnel syndrome ( $17 \%$ of patients with ATTR-CM vs $3 \%$ of patients with $\mathrm{HF})$, spinal stenosis (9\% vs $3 \%)$, hearing loss ( $11 \%$ vs $7 \%)$, and atrioventricular and left bundle branch block $(9 \%$ vs $5 \%$ ). Only the difference in carpal tunnel syndrome diagnosis was statistically significant. Half of all first carpal tunnel syndrome diagnoses occurred 6.7 or more years 


\begin{tabular}{|c|c|c|c|c|}
\hline Year & Newly included patients (n) & $\begin{array}{l}\text { Patients with prevalent } \\
\text { ATTR-CM* (n) }^{*} M^{*}\end{array}$ & Population at risk & $\begin{array}{l}\text { Prevalence per } \\
100000 t\end{array}$ \\
\hline 2008 & 96 & 96 & 9256347 & 1.0 \\
\hline 2009 & 69 & 141 & 9340682 & 1.5 \\
\hline 2010 & 74 & 183 & 9415570 & 1.9 \\
\hline 2011 & 57 & 204 & 9482855 & 2.2 \\
\hline 2012 & 97 & 259 & 9555893 & 2.7 \\
\hline 2013 & 83 & 291 & 9644864 & 3.0 \\
\hline 2014 & 92 & 332 & 9747355 & 3.4 \\
\hline 2015 & 100 & 382 & 9851017 & 3.9 \\
\hline 2016 & 122 & 443 & 9995153 & 4.4 \\
\hline 2017 & 117 & 499 & 10120242 & 4.9 \\
\hline 2018 & 87 & 510 & 10230185 & 5.0 \\
\hline
\end{tabular}

*The sum of patients included in previous years and still alive and newly included patients that year. First year of patient inclusion is 2008.

†Calculated as number of patients with prevalent ATTR-CM per year divided by population at risk.

ATTR-CM, transthyretin amyloid cardiomyopathy.

before the ATTR-CM diagnosis. Table 1 compares the disease history and the timing for potential red flags in the ATTR-CM cohort and the matched HF cohort.

The results based on the multivariate logistic regression model including the whole study population indicated that patients with a history of carpal tunnel syndrome, spinal stenosis, irritable bowel syndrome, supraventricular tachycardia, atrioventricular and left bundle branch block, or atrial fibrillation and flutter were more likely

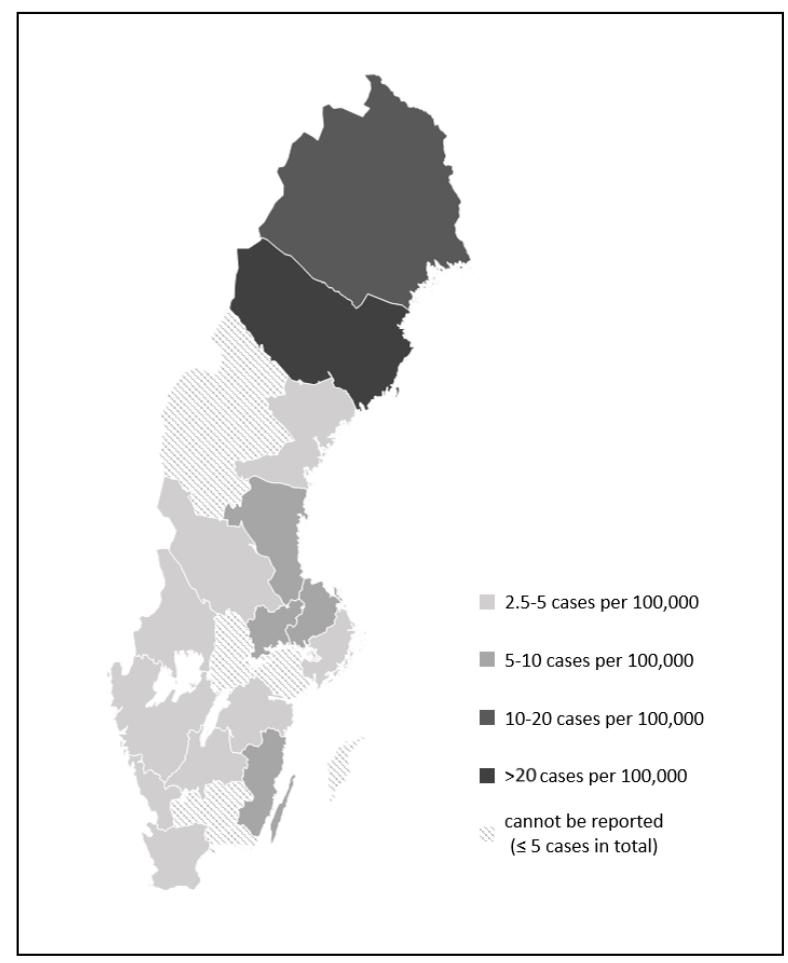

Figure 22018 prevalence in the Swedish regions. to be identified as patients with ATTR-CM. Specifically, patients with a history of carpal tunnel syndrome had an OR of 6.7 of being diagnosed with ATTR-CM. The model included all red flags and the results were controlled for patients' age, sex and comorbidity index. Table 4 lists the red flag diagnoses which demonstrated significant impact. The results for all covariates are found in online supplemental material.

\section{DISCUSSION}

This retrospective study combined multiple health registers covering the entire population of Sweden. Sweden is well known for high-quality health registers that are well designed for population studies. A total of 994 patients diagnosed with ATTR-CM were identified between 2008 and 2018. The mean age at diagnosis was 73 years and $30 \%$ were female. The prevalence of diagnosed ATTR-CM cases in 2018 was 5.0 per 100000 and the median survival was 37.6 months, with lower median survival in women (27.9 months) compared with men (43.5 months). Several red flags could be identified as early signs of ATTR-CM.

While the mean age is in line with previous studies, ${ }^{79-21}$ the proportion of female patients with ATTR-CM is higher than often suggested in the literature. A recent meta-analysis ${ }^{22}$ identified $13 \%$ of patients diagnosed with ATTR-CM as women. Results from a review of previous studies $^{23}$ demonstrated an average of $9 \%$ women among patients with ATTRwt-CM and 29\% among patients with ATTRv-CM. The $30 \%$ share of female patients with ATTR-CM found in this study likely reflects the relatively higher proportion of patients with ATTRv-CM in Sweden.

The prevalence of diagnosed ATTR-CM increased steadily over the study period to 5.0 per 100000 in 2018 . The lower numbers and the increase in prevalence in the 


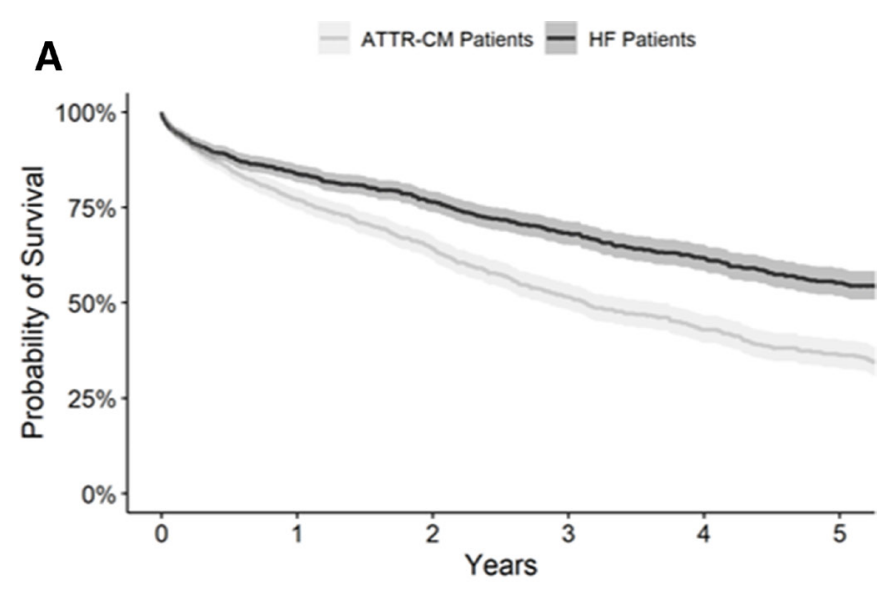

Patients at risk

$=994 \quad 696$

- $993 \quad 762$

$501 \quad 324$

223

155

612

464

355

272

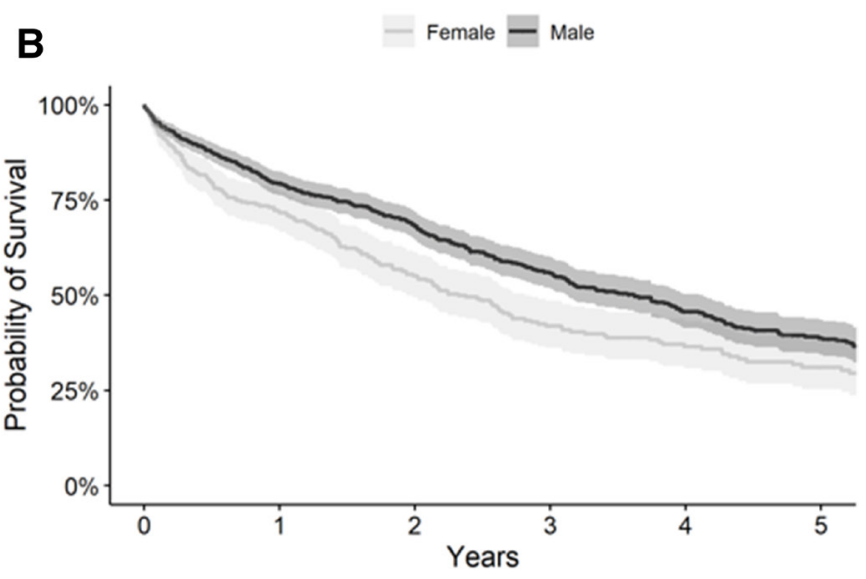

Patients at risk

$\begin{array}{rrrrrr}-297 & 196 & 131 & 83 & 60 & 42 \\ -\quad 697 & 500 & 370 & 241 & 163 & 113\end{array}$

Figure 3 Kaplan-Meier curve for overall survival of $(A)$ patients with ATTR-CM and $\mathrm{HF}$ and $(\mathrm{B})$ patients with ATTR$\mathrm{CM}$ by sex. ATTR-CM, transthyretin amyloid cardiomyopathy; $\mathrm{HF}$, heart failure.

earlier years are partly due to study design, with inclusion of patients starting in 2008. However, this default increase in prevalence likely affects only the first few years as many patients diagnosed before 2008 either died soon after or were identified and included in the population at a later specialist visit. The lower number of patients included during 2018 compared with prior years is also due to study design, as patients with an HF/CM diagnosis in 2018 and an AM diagnosis in 2019 or 2020 would have been assigned to 2018 but were not included in the study population due to end of data availability.

The observed rise in prevalent diagnosed cases over time likely reflects increased awareness of the disease as well as the use of non-invasive technologies for diagnosis of ATTR- CM, with higher numbers of patients
Table 4 Red flag diagnoses as predictors of ATTR-CM diagnosis (limited to red flags significant at the $5 \%$ level)

\begin{tabular}{|c|c|c|c|}
\hline Red flag diagnosis* & OR & $P$ value & $95 \% \mathrm{Cl}$ \\
\hline $\begin{array}{l}\text { Carpal tunnel syndrome } \\
\text { (unilateral and bilateral) }\end{array}$ & 6.718 & $<0.001$ & 5.542 to 8.143 \\
\hline Spinal stenosis & 2.702 & $<0.001$ & 2.092 to 3.489 \\
\hline Irritable bowel syndrome & 2.455 & 0.020 & 1.150 to 5.240 \\
\hline Supraventricular tachycardia & 1.897 & 0.024 & 1.087 to 3.309 \\
\hline $\begin{array}{l}\text { Atrioventricular and left } \\
\text { bundle branch block }\end{array}$ & 1.626 & 0.002 & 1.195 to 2.211 \\
\hline Atrial fibrillation and flutter & 1.214 & 0.008 & 1.053 to 1.400 \\
\hline \multicolumn{4}{|c|}{$\begin{array}{l}\text { 'The multivariate regression model included all red flags and the } \\
\text { results were controlled for patients' age, sex and comorbidity } \\
\text { index. The results for all covariates are found in online } \\
\text { supplemental material. } \\
\text { ATTR-CM, transthyretin amyloid cardiomyopathy. }\end{array}$} \\
\hline
\end{tabular}

diagnosed in the years following pivotal publications for such technologies. ${ }^{24}$ Still, underdiagnosis remains an important factor. Lindmark et $a l^{25}$ estimated the true prevalence of ATTRwt-CM in 2018 by specifically screening for undiagnosed ATTRwt-CM and estimated the prevalence of diagnosed and undiagnosed ATTRwt-CM at 16.6 per 100000 .

The median overall survival after ATTR-CM diagnosis in this study is similar to the survival after diagnosis reported in other studies, ${ }^{572026}$ and also survival in the HF cohort is aligned with previous results for Swedish patients with $\mathrm{HF}^{27}$ The higher mortality of patients with ATTR-CM compared with the matched HF cohort may indicate that ATTR-CM is a more severe and aggressive disease than 'garden variety' $\mathrm{HF}$ which, combined with lower disease awareness and delayed diagnosis, leads to poor survival outcomes.

The significantly shorter median survival estimated for women compared with men $(p=0.001)$ cannot be fully explained by observed patient characteristics. Instead, it may be a sign that women are diagnosed at a later stage of the disease compared with men. Kroi et $a l^{22}$ found that in studies which included autopsy for ATTR-CM identification, the share of female patients was generally higher than in studies which relied on diagnosis during lifetime, indicating that ATTR-CM in women is more often overlooked. This could be due to specific diagnostic challenges in women. Bruno et $a l^{23}$ argue that women, due to their smaller cardiac anatomy, show generally lower wall thickness than men and thus reach the generally suggested threshold for ATTR-CM diagnosis $(>12 \mathrm{~mm})$ later than men. Moreover, HF with preserved ejection fraction is more common in elderly women than elderly men, resulting in a lower degree of suspicion of ATTR-CM in this patient population. ${ }^{28}$

Comparing the occurrence of red flags in patients with ATTR-CM and in patients with HF is relevant from a clinical standpoint where patients with ATTR-CM need to be identified among patients who present 
with heart-related symptoms. We were able to confirm common red flags, ${ }^{6-12}$ but when compared with patients with HF, only carpal tunnel syndrome remained a significant red flag for ATTR-CM. Carpal tunnel syndrome and spinal stenosis occurred in substantial proportions of patients at a median of 6 years before diagnosis, potentially reflecting earlier manifestations of the disease process, while conduction blocks and atrial arrhythmias occurred closer to the time of diagnosis. However, about one-third of patients did not have a history of red flag diagnoses, and while red flags can help physicians in identifying ATTR-CM the lack of such early signals should not be used to exclude patients from further investigation.

This study has some limitations. First and most importantly, only diagnosed ATTR-CM cases could be identified in this study and underdiagnosis is frequent. Patients diagnosed with ATTR-CM were identified based on a combination of several ICD-10 codes since there is no final consensus on how to code the diagnoses of ATTRv-CM and ATTRwt-CM in Sweden. Some patients diagnosed with ATTR-CM may have been missed by the identification algorithm and some patients may have been falsely included. In addition, this study could not differentiate patients with and without biopsy-proven or otherwise clinically validated ATTR-CM diagnoses and it was not possible to differentiate ATTRv-CM and ATTRwt-CM cases. This is an important limitation as clinical presentations and prognoses differ between ATTRwt-CM and types of ATTRv-CM. ${ }^{29}{ }^{30}$ Finally, the exact date of the first ATTR-CM diagnosis was not known but was set to the first HF or CM diagnosis which fulfilled the inclusion criteria.

Despite these limitations, the carefully designed algorithm used to identify probable patients with ATTR$\mathrm{CM}$, and exclude patients with AL, was demonstrated by study results to work well. Prevalence rates, patient characteristics and mortality were in line with the existing literature. The higher prevalence rates found in Northern Sweden reflect the larger numbers of hereditary cases as well as the increased disease awareness of physicians in these regions, further increasing confidence in the method of patient identification used in this study. Moreover, the high-quality national health registers provide a reliant data source to study epidemiology and facilitated estimation of prevalence for the whole country without the need to extrapolate. Finally, the long follow-up period of up to 11 years leads to mature survival data and the long look-back period of a minimum of 10 years made it possible to study red flags that occurred several years before ATTR-CM diagnosis.

In conclusion, this study provides the first nationwide estimates of ATTR-CM prevalence and risk factors. The prevalence of diagnosed ATTR-CM cases increased over time and the median survival from ATTR-CM diagnosis was just over 3 years. This study revealed worse survival outcomes for women compared with men. The results reinforce the severity of the disease, high mortality and the importance of earlier diagnosis in order to effectively treat patients and prevent disease progression. This study provided supporting evidence about the importance of red flags and their potential in facilitating early diagnosis.

Author affiliations

${ }^{1}$ Quantify Research AB, Stockholm, Sweden

${ }^{2}$ Institute of Health and Society, University of Oslo Faculty of Medicine, Oslo, Norway

${ }^{3}$ Pfizer Innovations AB, Stockholm, Sweden

${ }^{4}$ Pfizer, Capelle a/d IJssel, The Netherlands

${ }^{5}$ Pfizer Denmark ApS, Ballerup, Denmark

${ }^{6}$ Pfizer Oy, Helsinki, Finland

${ }^{7}$ Department of Medicine and Clinical Research, Kuopio University Hospital and University of Eastern Finland, Kuopio, Finland

${ }^{8}$ Department of Cardiology, Oslo University Hospital Rikshospitalet, 0slo, Norway

${ }^{9}$ Department of Cardiology, Rigshospitalet, Copenhagen, Denmark

${ }^{10}$ Department of Cardiology, Wallenberg Center for Molecular Medicine and Lund University Diabetes Centre, Clinical Sciences, Lund University and Skåne University Hospital, Lund, Sweden

${ }^{11}$ The Wallenberg Laboratory/Department of Molecular and Clinical Medicine, Institute of Medicine, Gothenburg University and Sahlgrenska University Hospital, Gothenburg, Sweden

Contributors REL, JLH, CG, MHR and MV planned the work described in the article. REL and JLH were responsible for conducting and reporting the work. All authors contributed to refinement of the study protocol, interpretation and reporting of the results, and the final manuscript. REL is the guarantor.

Funding This study was sponsored by Pfizer.

Map disclaimer The inclusion of any map (including the depiction of any boundaries therein), or of any geographic or locational reference, does not imply the expression of any opinion whatsoever on the part of BMJ concerning the legal status of any country, territory, jurisdiction or area or of its authorities. Any such expression remains solely that of the relevant source and is not endorsed by BMJ. Maps are provided without any warranty of any kind, either express or implied.

Competing interests REL and JLH are employed by Quantify Research and funded by Pfizer to conduct this study; Quantify Research is a consultancy and works with a range of different pharmaceutical companies. CG, MHR, AMS and MV are Pfizer employees and hold Pfizer stock and/or stock options. JK received support from Pfizer for her collaboration in this manuscript, as well as grants or contracts from Sanofi, Pfizer and Amgen, Kuopio University Hospital, and Finnish Heart Research Foundation Academy of Finland. JK also received consulting fees and honoraria for lectures, presentations, speakers bureaus, manuscript writing or educational events from Sanofi, Pfizer, Amicus, Bayer, Amgen and MSD, as well as support for attending meetings from Sanofi, Pfizer, Amicus, Bayer, Amgen, MSD, Shire and Novo Nordisk. JK has received support for participation on a Data Safety Monitoring Board or Advisory Board from Amgen, Pfizer, Amicus and Sanofi. JK is also President (future/present/past) of the Finnish Society of Internists (2015-2021) and is the leader of the grant board at the Finnish Society of Medicine. EG has not received personal payments or support; his institution has received grants for work on this study and honoraria for lectures from Pfizer. FG received honoraria for his consulting services from Pfizer, Alnylam and lonis.

Patient consent for publication Not required.

Ethics approval Ethical approval for this study was obtained from the Swedish Ethical Review Agency in 2019.

Provenance and peer review Not commissioned; externally peer reviewed.

Data availability statement Data may be obtained from a third party and are not publicly available. All data were obtained from the Swedish National Board of Health and Welfare, the data holder of the national registers used in this study. Data can be obtained from the Swedish National Board of Health and Welfare upon approval from the Swedish Ethical Review Authority.

Supplemental material This content has been supplied by the author(s). It has not been vetted by BMJ Publishing Group Limited (BMJ) and may not have been peer-reviewed. Any opinions or recommendations discussed are solely those of the author(s) and are not endorsed by BMJ. BMJ disclaims all liability and responsibility arising from any reliance placed on the content. Where the content 
includes any translated material, BMJ does not warrant the accuracy and reliability of the translations (including but not limited to local regulations, clinical guidelines, terminology, drug names and drug dosages), and is not responsible for any error and/or omissions arising from translation and adaptation or otherwise.

Open access This is an open access article distributed in accordance with the Creative Commons Attribution Non Commercial (CC BY-NC 4.0) license, which permits others to distribute, remix, adapt, build upon this work non-commercially, and license their derivative works on different terms, provided the original work is properly cited, appropriate credit is given, any changes made indicated, and the use is non-commercial. See: http://creativecommons.org/licenses/by-nc/4.0/.

\section{ORCID iDs}

Rosa Elisabeth Lauppe http://orcid.org/0000-0002-5776-6600

Johan Liseth Hansen http://orcid.org/0000-0003-3954-8914

Johanna Kuusisto http://orcid.org/0000-0001-6550-3176

J Gustav Smith http://orcid.org/0000-0001-6285-9935

\section{REFERENCES}

1 Rubin J, Maurer MS. Cardiac amyloidosis: overlooked, underappreciated, and treatable. Annu Rev Med 2020;71:203-19.

2 Holmgren G, Costa PM, Andersson C, et al. Geographical distribution of TTR Met30 carriers in northern Sweden: discrepancy between carrier frequency and prevalence rate. J Med Genet 1994;31:351-4.

3 Suhr OB, Lindqvist P, Olofsson B-O, et al. Myocardial hypertrophy and function are related to age at onset in familial amyloidotic polyneuropathy. Amyloid 2006;13:154-9.

4 Lane T, Fontana M, Martinez-Naharro A, et al. Natural history, quality of life, and outcome in cardiac transthyretin amyloidosis. Circulation 2019;140:16-26.

5 Grogan M, Scott CG, Kyle RA, et al. Natural history of wild-type transthyretin cardiac amyloidosis and risk stratification using a novel staging system. J Am Coll Cardiol 2016;68:1014-20.

6 Ruberg FL, Grogan M, Hanna M, et al. Transthyretin amyloid cardiomyopathy: JACC state-of-the-art review. J Am Coll Cardiol 2019;73:2872-91.

7 Pinney JH, Whelan CJ, Petrie A, et al. Senile systemic amyloidosis: clinical features at presentation and outcome. J Am Heart Assoc 2013;2:e000098.

8 Yanagisawa A, Ueda M, Sueyoshi T, et al. Amyloid deposits derived from transthyretin in the ligamentum flavum as related to lumbar spinal canal stenosis. Mod Pathol 2015;28:201-7.

9 Maurer MS, Bokhari S, Damy T, et al. Expert consensus recommendations for the suspicion and diagnosis of transthyretin cardiac amyloidosis. Circ Heart Fail 2019;12:e006075.

10 Geller HI, Singh A, Alexander KM, et al. Association between ruptured distal biceps tendon and wild-type transthyretin cardiac amyloidosis. JAMA 2017;318:962-3.

11 Castaño A, Narotsky DL, Hamid N, et al. Unveiling transthyretin cardiac amyloidosis and its predictors among elderly patients with severe aortic stenosis undergoing transcatheter aortic valve replacement. Eur Heart J 2017;38:2879-87.

12 Huang J, Zhao S, Chen Z, et al. Contribution of electrocardiogram in the differentiation of cardiac amyloidosis and nonobstructive hypertrophic cardiomyopathy. Int Heart J 2015;56:522-6.
13 Ludvigsson JF, Andersson E, Ekbom A, et al. External review and validation of the Swedish national inpatient register. BMC Public Health 2011;11:450.

14 Wettermark B, Hammar N, Fored CM, et al. The new Swedish Prescribed Drug Register--opportunities for pharmacoepidemiological research and experience from the first six months. Pharmacoepidemiol Drug Saf 2007;16:726-35.

15 Brooke HL, Talbäck M, Hörnblad J, et al. The Swedish cause of death register. Eur J Epidemiol 2017;32:765-73.

16 Vela-Ojeda J, García-Ruiz Esparza MA, Padilla-González Y, et al. Multiple myeloma-associated amyloidosis is an independent highrisk prognostic factor. Ann Hematol 2009;88:59-66.

17 Elixhauser A, Steiner C, Harris DR, et al. Comorbidity measures for use with administrative data. Med Care 1998;36:8-27.

18 Garland A, Fransoo R, Olafson K. The epidemiology and outcomes of critical illness in Manitoba. Winnipeg, MB: Manitoba Centre for Health Policy, 2012.

19 Gilstrap LG, Dominici F, Wang Y, et al. Epidemiology of cardiac amyloidosis-associated heart failure hospitalizations among fee-forservice Medicare beneficiaries in the United States. Circ Heart Fail 2019;12:e005407.

20 Maurer MS, Elliott P, Comenzo R, et al. Addressing common questions encountered in the diagnosis and management of cardiac amyloidosis. Circulation 2017;135:1357-77.

21 Winburn I, Ishii T, Sumikawa T, et al. Estimating the prevalence of transthyretin amyloid cardiomyopathy in a large in-hospital database in Japan. Cardiol Ther 2019;8:297-316.

22 Kroi F, Fischer N, Gezin A, et al. Estimating the gender distribution of patients with wild-type transthyretin amyloid cardiomyopathy: a systematic review and meta-analysis. Cardiol Ther 2021;10:41-55.

23 Bruno M, Castaño A, Burton A, et al. Transthyretin amyloid cardiomyopathy in women: frequency, characteristics, and diagnostic challenges. Heart Fail Rev 2021;26:35-45.

24 Gillmore JD, Maurer MS, Falk RH, et al. Nonbiopsy diagnosis of cardiac transthyretin amyloidosis. Circulation 2016;133:2404-12.

25 Lindmark K, Pilebro B, Sundström T, et al. Prevalence of wild type transtyrethin cardiac amyloidosis in a heart failure clinic. ESC Heart Fail 2021;8:745-9.

26 Elliott P, Drachman BM, Gottlieb SS, et al. 1169 Interim analysis of data from a long-term, extension trial of tafamidis meglumine in patients with transthyretin amyloid cardiomyopathy. Eur Heart $J$ 2019;40.

27 Zarrinkoub R, Wettermark B, Wändell P, et al. The epidemiology of heart failure, based on data for 2.1 million inhabitants in Sweden. Eur $J$ Heart Fail 2013:15:995-1002.

28 Lam CSP, Donal E, Kraigher-Krainer E, et al. Epidemiology and clinical course of heart failure with preserved ejection fraction. Eur $J$ Heart Fail 2011;13:18-28.

29 Maurer MS, Hanna M, Grogan M, et al. Genotype and phenotype of transthyretin cardiac amyloidosis: THAOS (transthyretin amyloid outcome survey). J Am Coll Cardiol 2016;68:161-72.

30 Koike H, Tanaka F, Hashimoto R, et al. Natural history of transthyretin Val30Met familial amyloid polyneuropathy: analysis of late-onset cases from non-endemic areas. J Neurol Neurosurg Psychiatry 2012;83:152-8. 